Case Report

\title{
Fibroma - A misnomer : Case Series
}

\author{
Riya Verghese ${ }^{1}$, Amitha Ramesh ${ }^{2}$, Rahul Bhandary ${ }^{3}$, Biju Thomas ${ }^{4} \&$ Nishita L. Philip ${ }^{5}$ \\ ${ }^{1}$ Post Graduate, ${ }^{2,3}$ Professors, ${ }^{4}$ Professor \& HOD, ${ }^{5}$ Intern, Department of Periodontics, \\ A.B. Shetty M emorial Institute of Dental Sciences, Nitte University, M angalore, Karnataka, India. \\ Correspondence \\ Riya Verghese \\ Post Graduate, Department of Periodontics, A.B. Shetty M emorial Institute of Dental Sciences, \\ Nitte University, M angalore - 575 018, Karnataka, India. \\ Mobile : +9195914 59809 E-mail : riyamv999@gmail.com
}

\begin{abstract}
Objectives : Fibromas are proliferative fibrous lesions of the gingiva and oral mucosa that may cause esthetic and functional problems. Fibrous hyperplasia and Fibro-epithelial hyperplasia are histological variants of thesenon-neoplasticlesions
\end{abstract}

Methods: This article addresses the diagnosis, histological features and treatment of two cases of fibroma.

Results: These lesions are a result of trauma/ chronic irritation, or arise from cells of periodontium, periodontal ligament, or periosteum.

Conclusion : The cases demonstrate the need for proper diagnosis, role of biopsy and histologic evaluation in management of these lesions

Keywords: Fibrous hyperplasia, Fibroepithelial hyperplasia, inflammatory hyperplasia, neoplasia

\section{Introduction}

Fibromas are considered the most common benign soft tissue growth in the oral cavity. ${ }^{1,2}$ They may arise from the gingival connective tissue or from the periodontal ligament. They are slow- growing, spherical tumors that tend to be firm and nodular but may be soft and vascular. ${ }^{3}$ Epulis is a generic term used clinically to designate all discrete tumors and tumorlike masses of the gingiva. It serves to locate the tumor but not to describe it ${ }^{3}$

This lesion has a predilection for females, occurs in patients older than 30 years, is a few centimeters in diameter, pedunculated or sessile, and occurs frequently on the gingiva or buccal mucosa. The term, focal fibrous hyperplasia" as suggested by Daley et al.19905 which implies a reactive tissue response, is preferable to the term, fibroma" which implies incorrectly, a benign

Access this article online
Quick Response Code neoplastic proliferative fibrous connective tissue ${ }^{6}$.

The lesion presents as painless, sessile, round or ovoid, broad-based swelling, lighter in colour than surrounding tissue due to a reduced vascularity
${ }^{7}$.The surface may be ulcerated and diameter varies from 1 millimeter to several centimeters.Treatment of the ? broma involves surgical excision, and recurrences are very infrequent.

Irritation fibroma, or traumatic fibroma, is a common submucosal response to trauma from teeth or dental prostheses and was first reported in $1846^{11}$ as fibrous polyp and polypus

\section{CASE 1}

A 20 year old female patient reported to Department of Periodontics, A B Shetty Memorial Institute of Dental Sciences with the chief complaint of "swelling in upper right gum region" (Figure 1). History revealed a systemically healthy female with no family history of gingival epulis. The lesionwas firstnoticed 2 months ago and size remained constant since then. No history of dental and/or facial trauma was reported. The lesion was painless except on digital pressure. There was no history of bleeding except on digital pressure and use of interdental aids. Oral hygiene was found to be good with an $\mathrm{OHI}-\mathrm{S}$ score of 1.She had visted a private clinic 1.5 months ago and underwent a supragingival scaling.

\section{Examination of gingiva}

The lesion on the buccal gingiva was about sessile $8 *$ 
$7 \mathrm{mmin}$ size, pink in color, stippled on interdental papilla between maxillary lateral and canine. The lesion was mildly erythematous and firm in consistency. A radiograph of the area revealed no bony involvement (Figure 2).

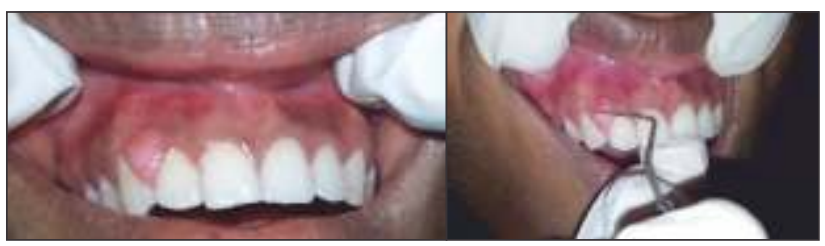

Figure 1a : clinical picture

Figure $1 \mathbf{b}$ : width

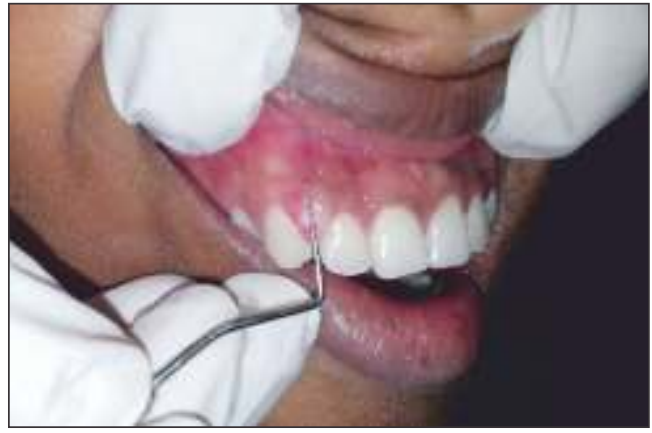

Figure $1 \mathrm{c}$ : length

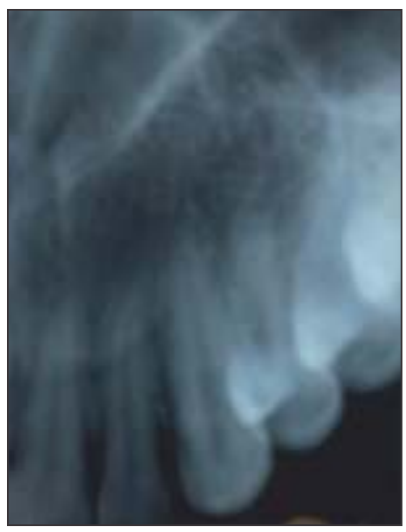

Figure 2 : Radiographic image.

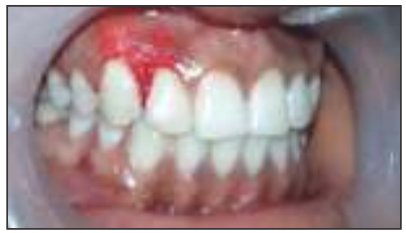

Figure 3 : Immediate post operative

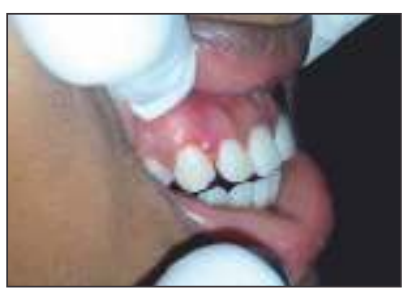

Figure 4 : Two-week postoperative pictures.

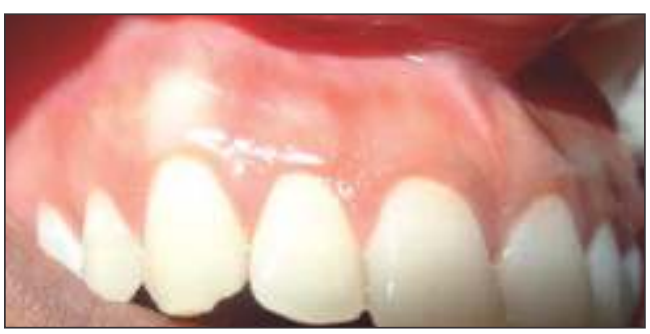

Figure 5 : 3 months follow-up

Phase I therapy with supragingival scaling was done. Patient was put on chlorhexidine mouthwash twice daily for 1 week. Then excision biopsy was performed using scalpel blade no 15 and Kirkland knife.Theexcised site were then finished with by abrasion method using a bur. Amoxycillin 500mg three times a day for 5 days and analgesics was prescribed to the patients post-operatively

On three months followup the area is healing well.No pain or discomfort and no di? culty in eating were reported. (Figure 5).

\section{Differential Diagnosis}

Irritaion fibroma, Peripheral giant cell granuloma, Peripheral ossifying fibroma,pyogenic granuloma, Papilloma, Gingival cyst were considered in differential diagnosis.

\section{Histopathology}

$H \& E$ stained section shows epithelium and connective tissue. Epithelium is parakeratinized stratified squamous epithelium with hyperplasia. Connective tissue shows collagen fibres, plump fibroblasts, chronic inflammatory cells, blood vessels and extravasated RBCs. (Fig $6 a \&$ Fig 6b)

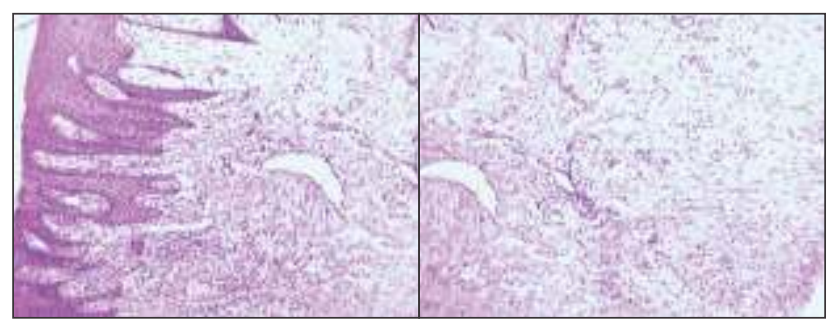

Figure 6a

Figure $6 b$

\section{CASE 2}

A 40 year old female patient reported to Department of Periodontics, A B Shetty Memorial Institute of Dental Sciences with the chief complaint of "swelling in lower left gum and lip region" (Figure 7). She had a history of hypertension and was on Anti-hypertensives with no family history of gingival epulis. The lesion was first noticed 1 year ago with constant fluctuations in size and episodes of rupture. No history of dental and/or facial trauma was reported. The lesion was painless except on digital pressure. Her oral hygiene was acceptable with an $\mathrm{OHI}-\mathrm{S}$ score of 2.6.She had not underwent any dental procedures in the past 1 year. 


\section{Examination of softtissues}

The lesion was about $1 * 1 \mathrm{~cm}$ in size, pink in color,in region of alveolar mucosa and labial mucosa of 33,34. The lesion was soft in consistency and mobile. A radiograph of the area revealed no bony involvement (Figure 8a).An occlusal radiograph was taken to check for calcified bodies. (Fig 8b)

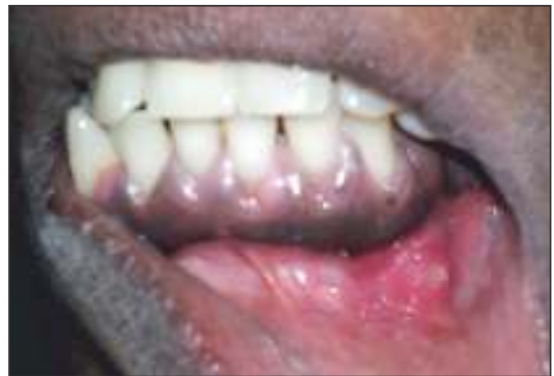

Figure 7 : clinical picture

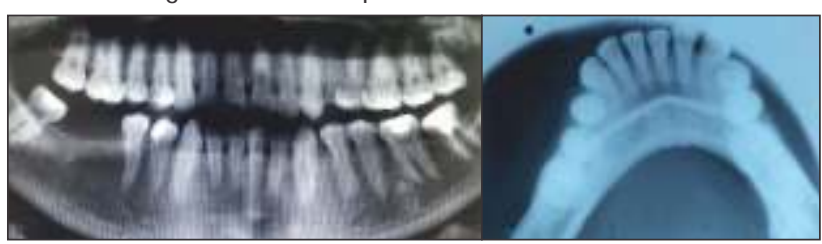

Figure 8a : Radiographic image-OPG Figure 8 b : Occlusal view shows absence of calicified bodies

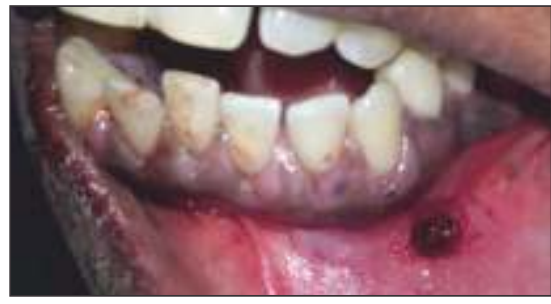

Figure 9 : Immediate post operative

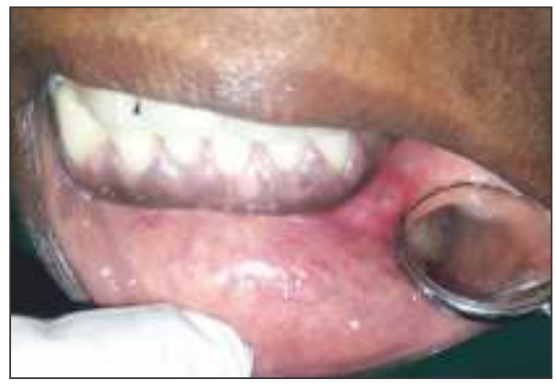

Figure 10 : Two-week postoperative picture

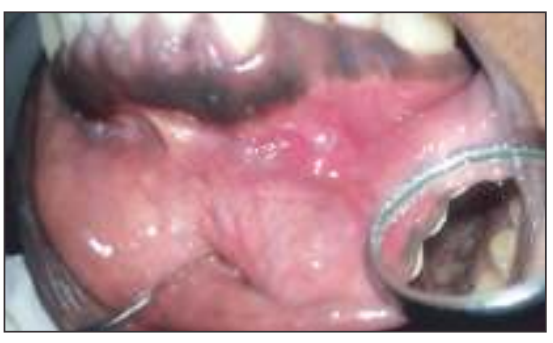

Phase I therapy with supragingival scaling was done and chlorhexidine mouthwash was prescribed twice daily for 1 week after which excision biopsy was performed using scalpel blade no 15 and 12. It was then cauterized with a ball elecrode.

Three month follow up the area is healing well.No pain or discomfort and no di? culty in eating were reported. (Figure 11).

\section{Differential Diagnosis}

Irritaion fibroma, Mucocele, Peripheral giant cell granuloma, Peripheral ossifying fibroma, pyogenic granuloma, Papilloma, were considered in differential diagnosis.

\section{Histopathology}

$H \& E$ stained sections show parakeratinized stratified squamous type of epithelium and connective tissue. Epithelium is hyperplastic. Connective tissue contains numerous blood vessels with some surrounded by plump and proliferating endothelial cells. Dense chronic inflammatory infiltrate comprising of lumphocytes, plasma cells and macrophages is present. Few areas show loosely arranged connective tissue. (Fig 12a \& 12b)

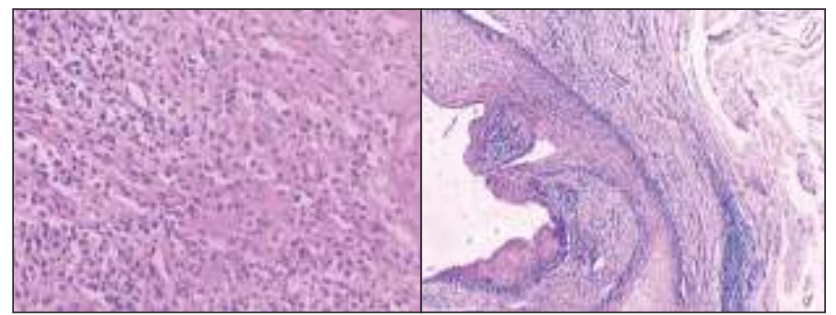

Figure 12 a

Figure $12 \mathrm{~b}$

\section{Discussion}

Tissue enlargement of the oral cavity often presents a diagnostic challenge because a diverse group of pathologic processes can produce such lesions. Within these lesions a group of reactive hyperplasia which develop in response to a chronic, recurring tissue injury stimulates an exuberant or excessive tissue repair response ${ }^{8}$

Hyperplastic reactive gingival/alveolar lesions including inflammatory gingival hyperplasia were the most common lesions according to a recent study ${ }^{9}$ 
Reactive gingival lesions have been classified into pyogenic granuloma, peripheral giant cell granuloma, fibrous hyperplasia and peripheral fibroma with calcification by Kfiret al ${ }^{10}$

As the most common nonneoplastic growth in the oral cavity, much has been written about the ?broma. It has been known as Irritation fibroma, Irritaional fibroma, Traumatic fibroma, Fibrous hyperplasia, Focal fibrous hyperplasia, Localized hyperplasia, Fibrous polyp, Fibroepithelial polyp ${ }^{11}$

The clinical presentation and epidemiology of most nonneoplastic growths in the oral cavity are quite similar; thus identi?cation is dependent on histopathological di? erentiation ${ }^{4}$. Histologically these lesions vary from granulation tissue to mature scar -like tissue, depending on age and vascularity. Lesions are collagenous, composed of mature fibrous tissue with prominent vascular pattern. Epithelial changes also correlate with the lesion's age and degree of inflammation. Fibro epithelial hyperplasias when inflamed are covered by uniformly hyperplastic epithelium, with arcading rete pattern when ulcerated Identification of any reactive hyperplastic gingival lesion requires the formulation of a differential diagnosis to enable accurate patient evaluation and management. These lesions must be separated clinically and histologically from precancerous, developmental and neoplastic lesions. Differential diagnoses include metastatic tumours in the oral cavity, angiosarcomas, gingival non- Hodgkin's lymphoma, Kaposi's sarcoma and haemangioma.

M ost of these lesions arise on gingiva, reflecting universal presence of inflammation in the interdental papillae which may be exaggerated by intensity of irritation, duration of lesion and metabolic effects of serum concentrations of hormones ${ }^{12}$ especially female hormones.

\section{References}

1. T. Esmeili, F. Lozada-Nur, and J. Epstein, "Common benign oral soft tissue masses," Dental Clinics of North America, vol. 49, no. 1, pp. 223-240, 2005.

2. Y. K? r, A. Buchner, and L. S. Hansen,"Reactive lesions of the gingiva. A clinicopathological study of 741 cases," J ournal of Periodontology, vol. 51, no. 11, pp. 655-661, 1980.
Fibro epithelial hyperplasia are reactive/ inflammatory conditions. These mucosal responses to chronic low grade irritation caused by plaque, and calculus or any other irritant $^{13}$

The term fibro-epithelial hyperplasia should not be confused with focal epithelial hyperplasia caused by HPV virus where all alterations occur in the epithelial layer of the mucosa with virtually no alteration in the underlying connective tissue. ${ }^{14}$

\section{Conclusion}

Connective tissue response to varied intensities of gingival irritation may be responsible for the myriad histological patterns observed in reactive hyperplasia This response may be influenced by the serum levels of certain endocrine hormones. However a substantial overlap may exist between the different histological entities .Distinction between hyperplasia and neoplasia needs to be clearly defined as neoplasias are not self- limiting conditions and long standing hyperplastic lesions in presence of chronic irritation can get converted to neoplasia. In addition to the physical characteristics of the lesion, the patient's demographics, presence of associated symptoms, related systemic disorders, and location and growth patterns of the lesion all give clues to adequately diagnose and treat their typical histopathologic architecture. Chances of transforming into neoplasia maybe high when lesion is of both epithelial and connective origin, as one of our cases is. Further studies are needed to confirm this hypothesis with longer follow up of the patients

\section{Acknowledgment}

The authors would like to thank Prof. (Dr.) Pushparaj Shetty (Head of Dept., Dept. of Oral Pathology) and Dr.Garima Jain, (Postgraduate student,Dept. of Oral Pathology) for histopathological analysis of the slides and providing us with the histopathological pictures of the slides

3. Carranza's Clinical Periodontology $11^{\text {th }}$ ed.

4. Vergotine. A Giant Cell Fibroma and Focal Fibrous Hyperplasia in a Young Child: A Case Report. Case reports in Dentistry.Volume 2012, Article ID 370242:1-5

5. Daley TD, Wysocki GP, Wysocki PD, Wysocki DM. The major epulides: Clinico pathological correlations. J Can Dent Assoc 1990; 56: 627-30 
6. Dieter Krahl, Andreas Altenburg, Christos C. Reactive hyperplasias, precancerous and malignant lesions of the oral mucosa. JDDG 2008; 6:217-232.

7. Ramzi Cotran, Vinay Kumar, Tucker Collins. Robbins Pathologic Basis of Disease, 6th Edition. W.B. Saunders.1999.

8. Mohammed Nazish Alam, S.C Chandrasekaran, Mohan Valiathan. Fibroma of the Gingiva: A Case Report of a 20 Year old Lesion. Int Journal of Contemporary Dentistry 2010; 1: 107-109.

9. Ramu S, Rodrigues C. Reactive hyperplastic lesions of gingiva- $A$ retrospective study of 260 cases. World Health Dentistry 2012;3(2): $126-130$
10. Kfir Y, Buchner A, Hansen LS. Reactive lesions of the gingiva. A clinicopathological study of 741 cases. J Periodontol 1980; 51:655-61

11. Gustafson B. A, Greenspan J. S. Multiple polypoid conditions of the oral mucosa. British J Oral Surgery 1974; 12, 91-95

12. NW Savage,CG Daly.Australian Dental Journal 2010; 55:(1 Suppl): 55-60.

13. Reddy V , Saxena S, Saxena S, Reddy M .Reactive hyperplasia of the oral cavity.J Clin Exp Dent. 2012;4(3):e136-40.

14. Madhusudan.A.S., Santosh Gupta, Sowmya.G.V. Focal Fibrous Hyperplasia: Report of two Cases.Int J Dental clinics2011; 3:111-112. 